\title{
The radiation belts of Jupiter and Saturn
}

\author{
E. Roussos ${ }^{1}$, P. Kollmann ${ }^{2}$ \\ ${ }^{1}$ Max Planck Institute for Solar System Research, 37077, Goettingen, Germany \\ ${ }^{2}$ Johns Hopkins University Applied Physics Laboratory, Laurel, MD 20723-6099, USA
}

Corresponding author: Elias Roussos, roussos@mps.mpg.de 


\begin{abstract}
The era of outer planet orbiters (Galileo, Juno and Cassini) is advancing our understanding of how the radiation belts of Jupiter and Saturn are structured, form and evolve well beyond what had been possible during the age of flyby missions and ground-based observations. The nearly two decades-long datasets of these missions, in the context of detailed and long-term observations of Earth's radiation belts, highlight which of the processes that accelerate particles to relativistic kinetic energies and limit their flux intensity can be considered more universal, and thus key for most extraterrestrial magnetospheres, and which reflect the unique aspects of each planet and its magnetospheric system. In this chapter we focus on the in-situ radiation belt observations in the context of theory, simulations and relevant measurements by Earth-based observatories. We describe both the average state and the time variations of Jupiter's and Saturn's radiation belts and associate them with specific physical processes.
\end{abstract}

\title{
1 Introduction
}

Similar to Earth, Jupiter and Saturn possess strong internal magnetic fields [Connerney et al., 2018; Dougherty et al., 2018] which react to the incoming solar wind flow and create large magnetospheres. These magnetospheres contain extended, stable, quasi-dipolar regions, one of the basic requirements for an efficient trapping and accumulation of high energy charged particles and thus the formation of radiation belts. On the other hand, Jupiter, Saturn and Earth differ in many other parameters.

In addition to being strongly magnetized, Jupiter and Saturn are fast rotating planets and with powerful plasma sources from active moons embedded within their magnetospheric boundaries. These three factors result in magnetospheres with absolute standoff magnetopause distances that are $\approx 60-99$ and $\approx 20-24$ times larger than Earth's, for Jupiter and Saturn respectively [Joy et al., 2002; Kanani et al., 2010]. Magnetospheric convection is mostly internally rather than solar wind driven [Kivelson, 2007]. The two planets' energetic particle trapping regions are very extended (Sections 3,4 ) and, especially at low L-shells and altitudes, negligibly disturbed by magnetospheric currents [Birmingham, 1982]. Furthermore, Jupiter's and Saturn's magnetic moment is oriented northward, resulting in energetic particle gradient and curvature drifts which are reverse in direction compared to those at Earth. Magnetic gradient and curvature electron drifts are opposite to the two planets' rotation and cancel out with the fast corotation drift at relativistic $(\mathrm{MeV})$ energies which are typical for radiation belts. At Earth this happens for much lower energy keV ions [Roederer, 1970; Thomsen and Van Allen, 1980]. The moons and rings contained within the magnetospheres can have a dual role: on the one hand they can supply the magnetosphere with plasma of nonionospheric or solar wind origin, which not only "inflates" them, but also drives waves that may accelerate particles to radiation belt energies (e.g. Shprits et al. [2018]). On the other hand, moons and rings can absorb particles and act simultaneously as particle sinks.

A key question is whether we can consider Earth's radiation belts as a prototype also for Jupiter and Saturn, or if the aforementioned differing aspects of those two planets' magnetospheres have a strong influence on how their radiation belts are structured and evolve. E.g. is the fast corotation of a magnetosphere important just for plasma or also for radiation belt particles? How strongly are the radiation belts of Jupiter and Saturn coupled to their host planets' unique characteristics, such as their moons and rings? To what extent can we probe the geophysics of these systems with radiation belt measurements?

A significant progress to answering such questions became possible only after Jupiter and Saturn were visited by dedicated orbiters (Galileo and Juno at Jupiter, Cassini at Saturn), even though the presence of radiation belts at the two planets was known ever since the detection of synchrotron emissions by Jupiter in 1959 [Drake and Hvatum, 1959] and the short duration Voyager and Pioneer flybys before 1981 (Sicard-Piet et al. [2011]; Krupp 
et al. [2016] and references therein). The short review that follows highlights observations by these three missions, and in particular in-situ measurements by several energetic particle instruments: the Energetic Particle Detector (EPD) and the Heavy Ion Counter (HIC) on Galileo [Williams et al., 1992; Garrard et al., 1992], the Magnetosphere Imaging Instrument (MIMI) on Cassini [Krimigis et al., 2004] and the Jupiter Energetic-particle Detector Instrument (JEDI) of Juno [Mauk et al., 2013]. The long term and continuous measurements of each mission with the same set of instruments mitigates calibration and time variability issues, a typical problem when comparing flyby datasets. Context from plasma, magnetic field and wave measurements (e.g. [Bagenal et al., 2016; Persoon et al., 2013; Meeks et al., 2016; Menietti et al., 2016]), Earth-based observations (e.g. Tsuchiya et al. [2011]; Yoshikawa et al. [2014]) and simulations (e.g. Santos-Costa et al. [2003]; Shprits et al. [2012]; Nénon et al. [2017]) are key for interpreting in-situ energetic particle measurements and are also discussed, although in less detail.

We start by listing a series of physical processes that are known to operate in Jupiter's and Saturn's radiation belts. Then, by describing each of the two belts' average state and temporal variations, we identify which of the previously listed processes are most important. Ion and electron belts have strong differences in both planets, and thus are discussed separately. In terms of energy, we distinguish the particle populations in two broad ranges above and below $\sim 1 \mathrm{MeV}$. References to energetic particles outside the radiation belt boundaries are also included, as these can be the belts' seed population. Discussions of time variability concern the radiation belts as a whole. E.g. a galilean satellite may be exposed to varying radiation belt environments, not necessarily because the belts change with time but because the tilted jovian magnetic field wobbles with respect to the moon's orbit. Such variations, which can be important for space weathering of planetary moons (e.g. [Milillo et al., 2016]), are not covered here.

\section{Physical processes}

\subsection{Particle sources and acceleration}

\subsubsection{Plasma sources}

Radiation belt particles (with the exception of particles from CRAND - see Sec. 2.1.2) start as low energy plasma that is stepwise accelerated to radiation belt energies (Sec. 2.1.32.1.4). A major plasma source at the Giant Planets is $\mathrm{SO}_{2}$ and $\mathrm{H}_{2} \mathrm{O}$ that originates from the active moons Io [Delamere and Bagenal, 2003; Kim and et al., 2018], Europa [Smyth and Marconi, 2006; Smith et al., 2019], and Enceladus [Cassidy and Johnson, 2010; DiFabio et al., 2011; Blanc et al., 2015; Wilson et al., 2017; Smith et al., 2018] and is in the following ionized and stepwise accelerated through its circulation in the magnetosphere (e.g. Kivelson [2006]; Rymer et al. [2008]). Other plasma sources are the inactive moons for example via sputtering and radiolysis [Jurac et al., 2001; Teolis et al., 2010], Titan's atmosphere, through thermal and hydrodynamic escape [Johnson et al., 2010], the rings [Christon et al., 2013; Elrod et al., 2014] via photolysis [Johnson et al., 2006], the planetary atmosphere through electron impact dissociation [Tseng et al., 2013], and the solar wind [DiFabio et al., 2011; Allen et al., 2018] that can enter through reconnection and Kelvin-Helmholtz instabilities at the magnetopause [Badman and Cowley, 2007; Delamere et al., 2013].

\subsubsection{CRAND}

CRAND (Cosmic Ray Albedo Neutron Decay) is a direct source of energetic protons and electrons for planetary radiation belts. Galactic cosmic rays (GCR), which are mostly protons at $\mathrm{GeV}$ energies [Shikaze et al., 2007] that are energetic enough to avoid deflection by the planetary magnetic field [Sauer, 1980; Kotova et al., 2018], can impact a planet's atmosphere or rings. The subsequent nuclear reactions create secondary neutrons [Hess et al., 1959; Cooper and Sturner, 2018] that decay back into protons at $\mathrm{MeV}$ to $\mathrm{GeV}$ energies, 
which can be magnetically trapped and populate the radiation belts. GCR protons impacting a hydrogen atmosphere, as that of Jupiter and Saturn, can also yield neutrons even though the hydrogen nucleus does not include them [Glass et al., 1977]. Neutron decay also produces $<1 \mathrm{MeV}$ electrons [Li et al., 2017] that receive energy both from the decay and the relativistic speed of the parent neutron. Only if the neutron spectrum has high fluxes at $\mathrm{GeV}$ energies, $\beta$-decay electron spectra may also reach $>\mathrm{MeV}$ energies.

Charged GCR secondaries (protons, alpha particles, or fragments of the nucleus) may become trapped on field lines connected to the region on which they originated and are thus expected to return to this region within one bounce period. Such secondaries can therefore only populate a radiation belt if the source material is tenuous enough to make follow-up losses inefficient [Roussos et al., 2018a].

\subsubsection{Radial Transport and adiabatic heating}

Diffusive or convective transport of electrons or ions into stronger magnetic fields leads to their adiabatic acceleration [Roederer, 1970]. Diffusive transport across small spatial scales requires processes that move particles randomly both inward and outward [Walt, 1994], such as ultra-low frequency (ULF) electric and magnetic field fluctuations (e.g. Khurana and Kivelson [1989]), variable magnetospheric convective flows (e.g. Murakami et al. [2016]), or changes of thermospheric winds [Brice and Mcdonough, 1973]. Many individual studies provide strong evidence of diffusion acting in both magnetospheres [Van Allen et al., 1980; Carbary et al., 1983; de Pater and Goertz, 1994; Roussos et al., 2007; Tsuchiya et al., 2011; Kita et al., 2015; Kollmann et al., 2017a].

Convective transport occurs on both global and local magnetospheric scales. Global scale convection exists in both magnetospheres [Barbosa and Kivelson, 1983; Ip and Goertz, 1983; Andriopoulou et al., 2012; Thomsen et al., 2012; Wilson et al., 2013]. On smaller scales, transport is effected through magnetospheric interchange (sometimes referred simply as "injections"). In such injections, charged particles get transported within few hours across many L-shells along flow channels that are few degrees wide in longitude [Mauk et al., 1999; Chen and Hill, 2008; Liu and Hill, 2012; Dumont et al., 2014; Paranicas et al., 2016]. Interchange is only efficient for $<1 \mathrm{MeV}$ particles, as higher energies drift quickly out of their flow channel [Paranicas et al., 2016] and no injection signatures have been observed at higher energies [Paranicas et al., 2010a; Clark et al., 2016]. Injections occur due to a centrifugal interchange instability that occurs at both Gas Giant planets due to their strong plasma sources and centrifugal force onn which this plasma is exposed to (Southwood and Kivelson [1987]; Kivelson [2006]). Interchange, which aims to even out flux tube content decreasing with increasing distance to the planet [Southwood and Kivelson, 1987], cannot be described well through diffusion, which smooths out any gradient in energetic particle phase space density [Schulz and Lanzerotti, 1974].

\subsubsection{Non-adiabatic acceleration}

Particles can be accelerated locally through field fluctuations with time scales on the order of the gyration time, which drive random energy losses or gains that can be described by energy diffusion [Schulz and Lanzerotti, 1974; Glauert and Horne, 2005; Woodfield et al., 2013]. A variety of processes can drive local acceleration, for example whistler mode chorus waves for electrons up to $\mathrm{MeV}$ [Woodfield et al., 2014; Menietti et al., 2014; de SoriaSantacruz et al., 2017]. The energy for accelerating particles will either be taken from the waves or from another particle population, for example at different pitch angle [Horne and Thorne, 2003]. Turbulence near reconnection sites can accelerate various ion species up to hundreds of $\mathrm{keV}$ [Radioti et al., 2007].

The auroral region is also able to accelerate particles to hundreds of $\mathrm{keV}$ and $\mathrm{MeV}$ energies [McKibben et al., 1993; Roussos et al., 2016; Palmaerts et al., 2016; Clark et al., 
2017; Paranicas et al., 2018; Mauk et al., 2018; Clark et al., 2018]. However, these particles are field-aligned, often to the extent that they will be absorbed in the opposite hemisphere. If instead they scatter and become trapped (e.g. Speiser [1965]; Young et al. [2008]; Roussos et al. [2016]) they can be further accelerated by radial inward transport.

\subsection{Particle sinks and deceleration}

\subsubsection{Moon absorption}

The Giant Planets have moons orbiting within the radiation belts which absorb energetic particles hitting their surface. To first order, the lifetime of a particle against moon absorption equals the successive encounter time between the moon and the particle and can be very large for electrons that drift with the same speed as a moon orbits around a planet. These are electrons in the $\mathrm{MeV}$ range for Saturn and tens of $\mathrm{MeV}$ for Jupiter. The encounter probability is reduced if the paths of the moon and particle are not matching due eccentric moon orbits, non-circular particle drifts, or tilts between magnetic and orbit plane. In addition, there is a probability that the charged particle would evade moon absorption due to its gyration, bounce and drift motions, or due to its deflection at field distortions in the local moon-magnetosphere interaction region. For all these reasons, moon absorption probabilities have strong dependencies from energy, species and charged state [Thomsen and Van Allen, 1980; Hood, 1983; Santos-Costa and Bourdarie, 2001; Selesnick and Cohen, 2009; Krupp et al., 2012].

\subsubsection{Charge exchange}

Singly charged ions $I^{+}$can undergo charge exchange with another particle $X: I^{+}+$ $X^{x} \rightarrow I+X^{x+1}$. The cross sections for charge exchange are usually larger for neutral particles $(x=0)$ than for positive ions [Fujiwara, 1976]. The energetic ion $I^{+}$turns into an energetic neutral atom $I$ (ENA) that is not trapped in the magnetic field anymore and usually escapes the magnetosphere. Charge exchange is therefore a loss process for singly charged ions. In rare cases, the ENA may re-ionize before leaving the magnetosphere, for example when grazing the planet's atmosphere, and become a source for low altitude particle radiation [Krimigis et al., 2005]. Multiply charged ions [Clark et al., 2016; Selesnick and Cohen, 2009] can also be affected by charge exchange [Fujiwara, 1976], but the process only changes their charge state (that many radiation instruments do not distinguish) while keeping the resulting particle trapped.

\subsubsection{Energy loss}

Energy loss can become equivalent to a particle loss in the context of measurements at a fixed energy range, as it is typical for charged particle detectors [Schulz and Lanzerotti, 1974]. Still, when there are many particles at high energies (hard or rising energy spectrum), these can supply the lower energies, turning the energy loss process at some energies into a source for some others. There are several means how a charged particle can lose energy at Jupiter and Saturn: energy loss due to ionization while it passes through neutral gas or ring grains [Kollmann et al., 2013; Kollmann et al., 2015], while it passes through plasma due to collisions with free electrons [Nénon et al., 2018], or while being deflected in strong magnetic fields due to synchrotron emission [Santos-Costa and Bourdarie, 2001].

\subsubsection{Pitch angle scattering and friction}

Various processes may lead trapped particles into the loss cone, reaching into the increasingly dense layers of the exosphere and atmosphere of a planet, where they are absorbed. The change in pitch angle can occur continuously (pitch angle friction) or randomly (scattering). Friction occurs along with synchrotron emission [Santos-Costa and Bolton, 2008]. 


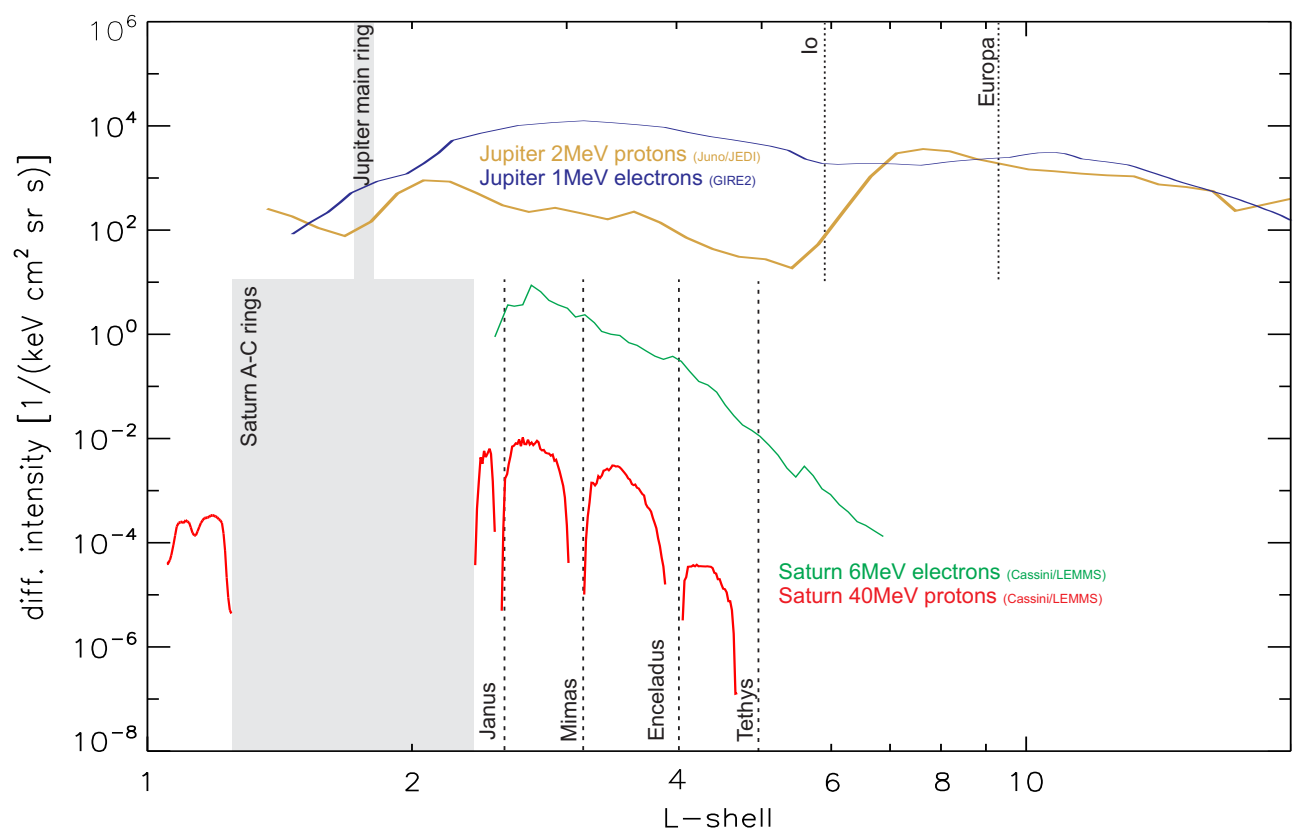

Figure 1. Sample intensity profiles of the radiation belts of Jupiter and Saturn as a function of equatorial distance. Saturn measurements outside of rings: average over Cassini mission [Kollmann et al., 2018a], Saturn protons inward of rings: corrected data [Roussos et al., 2018b], Jupiter protons: measurements from 2018 DOY 38 (Juno), Jupiter electrons: GIRE2 data compilation [de Soria-Santacruz et al., 2016]. A minor proton belt component slightly outwards of Saturn's main rings [Buratti et al., 2018] is not visible on this scale.

Scattering has many origins and is usually described through pitch angle diffusion [Schulz and Lanzerotti, 1974; Woodfield et al., 2013]. Drivers are wave-particle interactions, for example whistler mode hiss [Glauert et al., 2014; Nénon et al., 2017] and chorus [Shprits et al., 2012; de Soria-Santacruz et al., 2017] for electrons and electromagnetic ion cyclotron (EMIC) waves for ions [Nénon et al., 2018]. Such waves can arise from particle anisotropies [Kennel and Petschek, 1966], as they can result from radial transport [Bolton et al., 1997] or ion pickup [Huddleston et al., 1998]. High radiation intensities can also drive the growth of such waves so that radiation intensities are therefore self-limited to the Kennel-Petschek limit [Kennel and Petschek, 1966; Summers et al., 2011; Mauk, 2014], at least in the absence of strong sources.

Pitch angle diffusion can also result from scattering in matter [Santos-Costa and Bourdarie, 2001]. Such scattering is most important for electrons or $\lesssim 0.5 \mathrm{MeV}$ ions. Heavy and/or energetic ions do not scatter much even when most of their energy is lost [Kollmann et al., 2013; Nenon et al., 2018].

\section{Saturn's radiation belts}

\subsection{Average configuration}

\subsubsection{Ions}

Saturn's permanent ion radiation belt (for energies $>10 \mathrm{MeV}$, where $\mathrm{MeV}$ measurements are most reliable) extends from close to Saturn's visible surface $(L \approx 1.03)$ until the orbit of the moon Tethys $(L=4.9)$ [van Allen et al., 1980; Kollmann et al., 2013; Roussos et al., 2018b]. This belt is segmented into six sectors bound between the planet, the dense (A-C) rings and the orbits of the moons Prometheus/Pandora, Janus/Epimetheus, Mimas, 
Enceladus and Tethys (Fig. 1). Each sector is isolated from the other through moon proton absorption, while Tethys restricts ion transport between the belts and the magnetosphere [Roussos et al., 2008]. This restriction is the reason protons dominate the ion composition at these energies and L-shells, even though the most abundant ion species in the rest of magnetosphere and below $1 \mathrm{MeV}$ are $\mathrm{H}_{0-3} \mathrm{O}^{+}$[Sergis et al., 2007; Wilson et al., 2017]. Upper limit intensities of heavier ions in the $>10 \mathrm{MeV} /$ nuc energy range are at least an order of magnitude lower than those of protons [Armstrong et al., 2009].

The primary source of these belts is CRAND. CRAND protons may come from both Saturn's rings and atmosphere for $\mathrm{L}>2.27$. Inward of the rings $(\mathrm{L}<1.22)$ ring CRAND definately dominates (Cooper [1983]; Roussos et al. [2018b], Sec. 2.1.2, Fig. 2A). While moons and dense rings absorb CRAND protons along their orbits (Sec. 2.2.1, Fig. 2B), tenuous material from Saturn's exosphere and its D- and G- rings reduce the proton intensities only partially (Roussos et al. [2018b]; Kollmann et al. [2018b], Fig. 2C). The Enceladus generated E-ring and gas torus do not have a strong impact on the proton intensities [Kollmann et al., 2013].

For $\mathrm{L}>2.27$ radial diffusion (Sec. 2.1.3) acts as an additional loss mechanism, since it slowly drives CRAND protons into the moon orbits, regulating the rate at which they get absorbed. This process is also responsible for a broadening of proton depleted regions outside of the moon L-shells. The radial diffusion coefficient increases with distance, causing enhanced losses and lower proton intensities in the outer radiation belts (Kollmann et al. [2013], Fig. 2D).

Several ion belt components exist also in the $\mathrm{keV}$ energy range. At low altitudes $(\mathrm{L}<$ 1.06), stripped ENAs (Sec. 2.2.2) are a source of a weak intensity belt comprising tens of $\mathrm{keV}$ ions [Krimigis et al., 2005; Roussos et al., 2018b; Krupp et al., 2018]. Outside of the $\mathrm{MeV}$ proton belts $(\mathrm{L}>4.9), \mathrm{keV}$ energetic ion fluxes peak at $6 \lesssim L \lesssim 9$, depending on energy [Kollmann et al., 2011]. The intensities decrease from the peak toward $\mathrm{L} \approx 5$, potentially due to charge exchange with the Enceladus neutral torus [Paranicas et al., 2008; Dialynas et al., 2009]. This ion population rarely penetrates inward of $L=4$ [Roussos et al., 2018c] and has no sharp outer boundary [Allen et al., 2018].

\subsubsection{Electrons}

Saturn's electron radiation belt extends outward of Saturn's A-ring $(L>2.27)$ and peaks at $\mathrm{L} \approx 2.5$ (Fig. 1). It does not have a sharp outer boundary at a moon orbit, but decreases with increasing L. Measurable intensities of trapped, $\mathrm{MeV}$ electrons typically cease outward of $\mathrm{L} \approx 7$ [Roussos et al., 2014]. The belt's seed population is in the middle magnetosphere (ring current, $\mathrm{L} \approx 8-12$ ), from where electrons get accelerated adiabatically at least down to $\mathrm{L}=4$, where high intensities are found up to $\approx 1 \mathrm{MeV}$. For reference, significant intensities can be found up to several $10 \mathrm{MeV}$ at Jupiter [Kollmann et al., 2018a]. About 50\% of the seed electrons are field aligned [Clark et al., 2014], suggesting that they may be partly maintained through acceleration in the auroral region (Sec. 2.1.4).

Local acceleration through Whistler mode chorus waves (Sec. 2.1.4) is expected to be inefficient [Shprits et al., 2012] even though the intensity of these waves is significant [Menietti et al., 2014]. Radiation belt electrons are mainly lost through scattering (Sec. 2.2.4) in neutral material [Lorenzato et al., 2012]. Electrons are lost to moons and ring arcs [Hedman et al., 2007; Andriopoulou et al., 2012] but the resulting wakes along the electron drift direction are refilled via radial diffusion quicker than the electron-moon re-encounter time [Van Allen et al., 1980; Roussos et al., 2007], explaining why electron belts are not segmented as the proton ones at the moon orbits. Long re-encounter times result from electron drifts that are sensitive to global convective flows (Sect. 2.1.3) and which distort the shape of their nominally circular drift electron orbits, limiting their residence time along the circular sweeping corridors of moons and rings [Roussos et al., 2016]. The same flows are also responsible for persistent local time asymmetries in the spectra of energetic electrons, mostly 

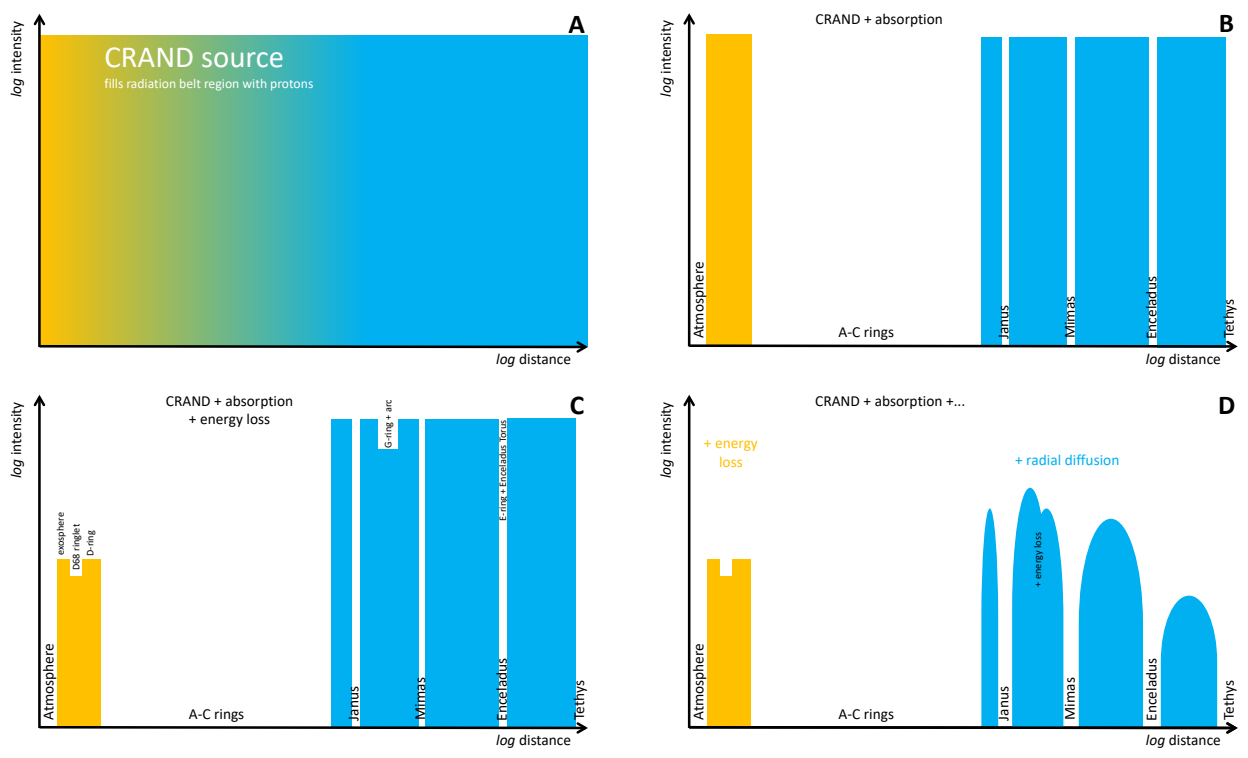

Figure 2. Sketch on how different processes shape the radial distribution of Saturn's proton belts (see red curve in Fig. 1). Panel A: CRAND provides the protons (Sec. 2.1.2). Panel B: Moons and dense rings are responsible for the separation of the belts (Sec. 2.2.1, 2.2.3). Panel C: Energy loss and absorption in tenuous material changes the shape of the belts (Sec. 2.2.3). Panel D: Radial diffusion smooths out the edges of the belts (Sec. 2.1.3).

along the noon-midnight direction [Carbary et al., 2009; Paranicas et al., 2010b; Thomsen et al., 2012; Andriopoulou et al., 2013], and the presence of local-time confined belt components (termed "microbelts") at the outer edge of Saturn's A-ring [Roussos et al., 2018a].

No energetic electrons were observed close to the planet $(\mathrm{L}<1.22)$ [Roussos et al., 2018b], consistent with the lack of synchrotron emissions from that region [Grießmeier et al., 2011]. Saturn's main rings block inward transport of electrons coming from beyond the rings to those low L-shells. Local sources like CRAND (Sec. 2.1.2), are not fast enough compared to local electron losses to dust and neutrals (Sec. 2.2.3, 2.2.4).

\subsection{Time variability}

\subsubsection{Ions}

The most striking change in Saturn's MeV proton belts is their transient extension beyond their nominal outer boundary at $\mathrm{L}=4.9$ [Roussos et al., 2008]. Such extensions commence when $\mathrm{MeV}$ ions of Interplanetary Coronal Mass Ejections (ICME), which penetrate easily down to $\mathrm{L} \approx 10$ [Kotova, 2016], get rapidly transported towards $\mathrm{L} \approx 5$ due to magnetospheric convection induced by the passage of an ICME shock. This process forms transient ion belts which have been observed to last between 3 and 9 weeks. These are the only events known that can induce a fast, global transport of $\mathrm{keV}$ ions into low L-shells $(\mathrm{L} \approx 4)$, before charge exchange in the Enceladus neutral cloud depletes them [Roussos et al., 2018d]. Transient, local $<1 \mathrm{MeV}$ ion flux enhancements from interchange injections (Sec. 2.1.3) are rarely observed inward of $\mathrm{L} \approx 5$ and are limited below few hundred $\mathrm{keV}$.

In comparison, changes in the permanent proton belts are marginal, but have a wellresolved profile (Fig. 3, panels a-c). A slow, steady increase of proton fluxes resulting from the variability of the CRAND source [Roussos et al., 2011] is balanced by abrupt flux dropouts 

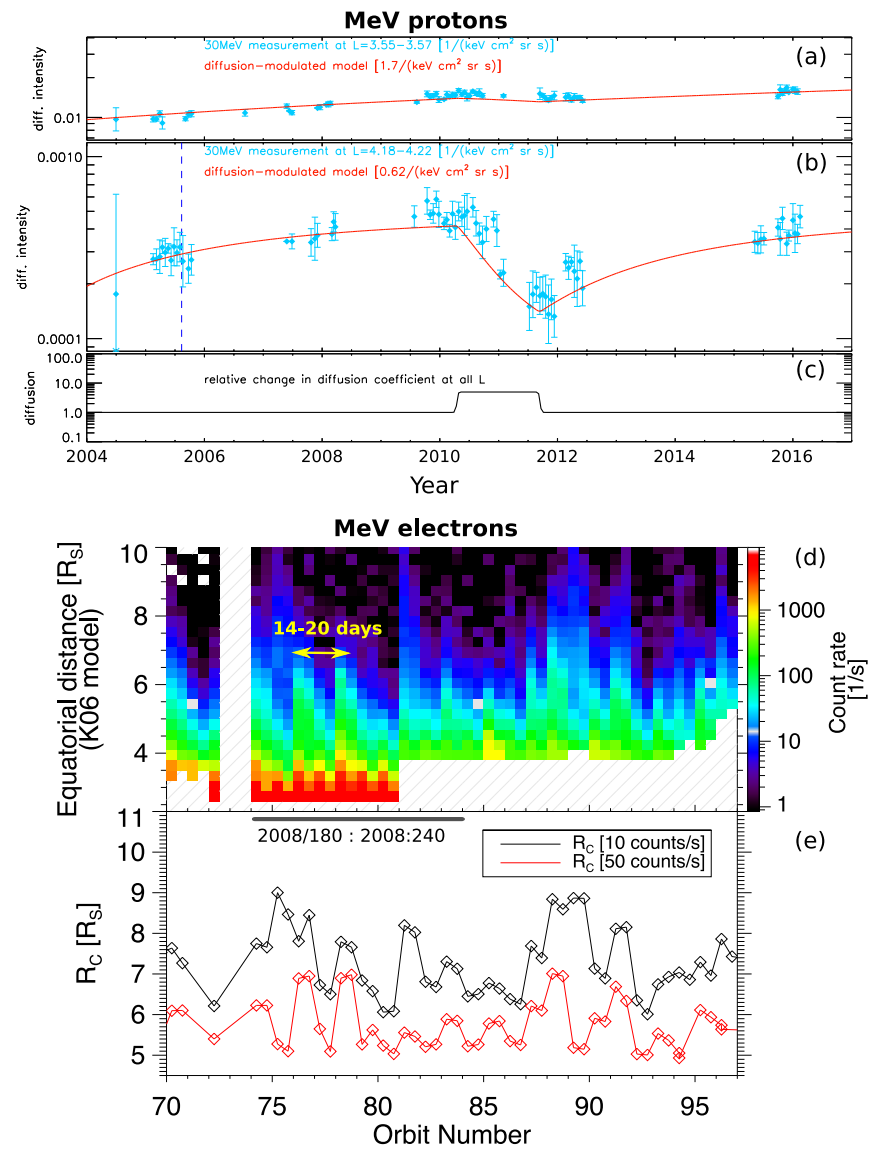

Figure 3. MeV proton and electron variability in Saturn's radiation belts. Panels (a) to (b) show long term changes in $\approx 30 \mathrm{MeV}$ proton fluxes, for two different L-shells. The red curve shows a simulated profile assuming a time-dependent radial diffusion coefficient (c). Panel (d) shows an orbit-distance spectrogram of $>1$ $\mathrm{MeV}$ electron count-rates at Saturn, while panel (e) the corresponding electron belt extension, $R_{C}$, for two count-rate thresholds, for a period in 2008. A 14-day periodicity is highlighted in yellow, and a similar period was simultaneously observed in the solar wind (grey bar). Panels (a)-(c) are based on Kollmann et al. [2017a], (d)-(e) on Roussos et al. [2018c].

over time scales of 1-2 years resulting from variable magnetospheric diffusion modulating the proton loss rates [Kollmann et al., 2017a].

\subsubsection{Electrons}

In stark contrast to the proton belts, changes in electron radiation belts are rapid and large in amplitude, affecting their intensity and L-shell profile. The reason is that communication of the electron belts with their highly variable source region in the ring current is not restricted by Saturn's moons [Roussos et al., 2014]. This variability has multiple sources. Biweekly oscillations of the belts all the way down to A-ring indicate that Corotating Interaction Regions (CIRs) in the heliopshere influence the electron belts (Fig. 3, panels d-e). Transient electron radiation belts can also follow ICMEs or large scale injections in the magnetotail [Roussos et al., 2018c]. 
Transients typically evolve on time scales faster than 2-3 weeks, much faster than for protons. This difference suggests that electron transport is dominated by variable convection, not stochastic radial diffusion. E.g. CIRs modulate the strength of magnetospheric convection, which in turn controls the radial transport of energetic electrons [Roussos et al., $2018 \mathrm{c}$,a]. Fast. but localized changes in the electron radiation belts have been observed also in the keV energy range. Such electrons penetrate to small L-shells and sustain the radiation primarily through interchange [Paranicas et al., 2010a]. Tail reconnection can trigger periods of deeply penetrating, recurring interchange carrying keV electrons to low L-shells faster than 10-20 hours [Thomsen et al., 2016].

Not all aspects of the electron belts' variability have been explained. In particular, a 2-3 month period in 2011, when the electron belts were persistently low in intensity, has no clear explanation.

\section{Jupiter's radiation belts}

\subsection{Average configuration}

\subsubsection{Ions}

The inner edge of Jupiter's ion belts is limited by the region of stable magnetic trapping at about $1.2 R_{J}$ [Nenon et al., 2018]. Jupiter's ion radiation belts do not have a clear outer boundary but gradually decrease in intensity outward (Fig. 1). Different to Saturn, they are less clearly separated by moon orbits. This is because the non-alignment between the magnetic equatorial and the orbital plane of the moons makes ion-moon encounters less likely than at Saturn. This explains why ions of both iogenic and solar wind origin (e.g. helium, oxygen, sulphur at various charge states) exist across the magnetosphere [Cohen et al., 2001; Selesnick and Cohen, 2009].

Europa and its gas torus only have a minor impact on the outermost radiation belt [Lagg et al., 2003; Kollmann et al., 2016]. The highest ion intensities at hundreds of keV are observed in this outermost belt that peaks at $\mathrm{L} \approx 7$ [Mauk et al., 2004; Kollmann et al., 2018a]. Ionization of material released from Io and subsequent pickup leads to the generation of EMIC waves, that result in pitch angle diffusion, scattering of ions into the atmosphere [Nénon et al., 2018], and eventually a strong depletion in ion intensities near the moon's L-shell $(L=5.9)$ that forms the inner edge of the outer ion belt (Thomsen et al. [1977], Fig. 1). Still, some ions can be transported across Io's orbit and form another radiation belt peaking at $L \approx 3$ [Garrett et al., 2015; Nénon et al., 2018]. The next radiation belt segment peaks at $L \approx 2$ and extends until Jupiter's main ring $(L=1.8)$. This is the belt with the highest ion energies $(>350 \mathrm{MeV})[$ Pehlke, 2000]. The best ion measurements are in helium, suggesting a source from spallation of ring material [Fischer et al., 1996]. Given that the abundance of other species is currently undetermined, contributions from radially transported magnetospheric ions is not excluded [Nenon et al., 2018]. The innermost radiation belt $(L<2)$ is populated with such magnetospheric ions having energies of at least $1 \mathrm{MeV}$. Since outward of that belt intensities of such ions are low, it was suggested that it is sourced by stripped energetic neutral atoms rather than diffusive transport [Kollmann et al., 2017b].

\subsubsection{Electrons}

Electron intensities around Jupiter are not clearly separated by location, so that it can be said that Jupiter has a single electron radiation belt. The intensities in its core are so high that they are often outside of the specifications that particle radiation measuring instruments were designed for. Therefore there exist relatively few high-quality measurements and inferences of intensities therefore rely on heavily corrected data from particle instruments, scarce measurements from flyby missions, synchrotron emissions, and particle radiation responses 
of imaging instruments [Taherion et al., 2008; de Soria-Santacruz et al., 2016; Becker et al., 2017; Nénon et al., 2017].

The seed population of the electron belt is in the outer magnetosphere. Evidence that adiabatic radial transport is a key energy source for $\mathrm{MeV}$ electrons down to at least $\mathrm{L} \approx 20$ has been presented by [Kollmann et al., 2018a]. Seed electrons that can be adiabatically heated are provided by a variety of processes, such as auroral acceleration [Tomás et al., 2004]. Whistler mode chorus waves [Menietti et al., 2016] are thought to provide additional acceleration on freshly injected particles [Woodfield et al., 2013] but become inefficient after the distributions reach a steady-state [de Soria-Santacruz et al., 2017]. Pitch angle diffusion limits the electron intensities below the Kennel-Petschek limit, at least around $\mathrm{L}=8$ [Mauk and Fox, 2010].

Electron intensities drop by a factor of $<10$ at $L \approx 6$, because it is thought that they are absorbed by Io, except at energies that have similar drift speeds as the moon's orbital speed [Santos-Costa and Bourdarie, 2001]. The absorption losses are enhanced by pitch angle diffusion [Nénon et al., 2017]. MeV electron intensities peak at $L \approx 3$ (de SoriaSantacruz et al. [2016], Fig. 1). Significant electron intensities at Jupiter exist up to several tens of $\mathrm{MeV}$, as synchrotron emissions indicate and simulations predict [de Pater and Goertz, 1994; Nénon et al., 2017].

\subsection{Time variability}

Variability in Jupiter's radiation belts is less explored with in-situ data compared to Saturn, primarily due to the long orbit periods of Galileo and Juno, and the spatial dependencies on planetary SIII longitude [Seidelmann and Divine, 1977] that are difficult to disentangle from L-shell and magnetic local time.

\subsubsection{Ions}

Energetic ions at Jupiter appear much more variable than at Saturn [Paranicas et al., 1999, 2002; Mauk et al., 1999; Selesnick et al., 2001a] but the time scales have not yet been explored. Given that the Galilean moons cannot prevent ion transport, it is expected that variability would develop faster than the year-long changes of Saturn's proton belts and may reflect the rate of radial ion transport and acceleration in the system, estimated to be in the range of weeks to months [Cohen et al., 2001]. Transient acceleration events producing MeV ions have been observed to occur at 25-30 $\mathrm{R}_{\mathrm{J}}$ [Selesnick et al., 2001b] but their origin is unclear. At lower keV energies, radiation belt ions may be sustained by dipolarization events, which recur every few days, and interchange injections which could be modulated by the aforementioned dipolarizations [Mauk et al., 1999; Kronberg et al., 2007; Dumont et al., 2014; Louarn et al., 2014]. The same time scale may then apply for the low-altitude ion belt [Kollmann et al., 2017b], if it is indeed produced by stripping of ENAs generated at the tori of Io and Europa.

\subsubsection{Electrons}

Variability of the jovian $\mathrm{MeV}$ electron belt can be observed remotely through the synchrotron radiation it emits, which reveals that diffusive transport from the electron source region ( $\mathrm{L}>6$ ) to its innermost regions lasts about 2 years [de Pater and Goertz, 1994]. Longterm changes in this belt have been positively correlated to variable solar wind parameters, with time lags of 0.7 or 2.7 years [Santos-Costa et al., 2008; Galopeau and Gérard, 2001].

Many studies identified additional short or medium-term changes in the synchrotron emissions (e.g. Miyoshi et al. [1999]). One of those was linked to the entry of the disrupted comet Shoemaker-Levy 9 (SL9) in Jupiter's magnetosphere and its impact on the planet, with various mechanisms, such as enhanced chorus emissions or shock-induced acceleration, con- 

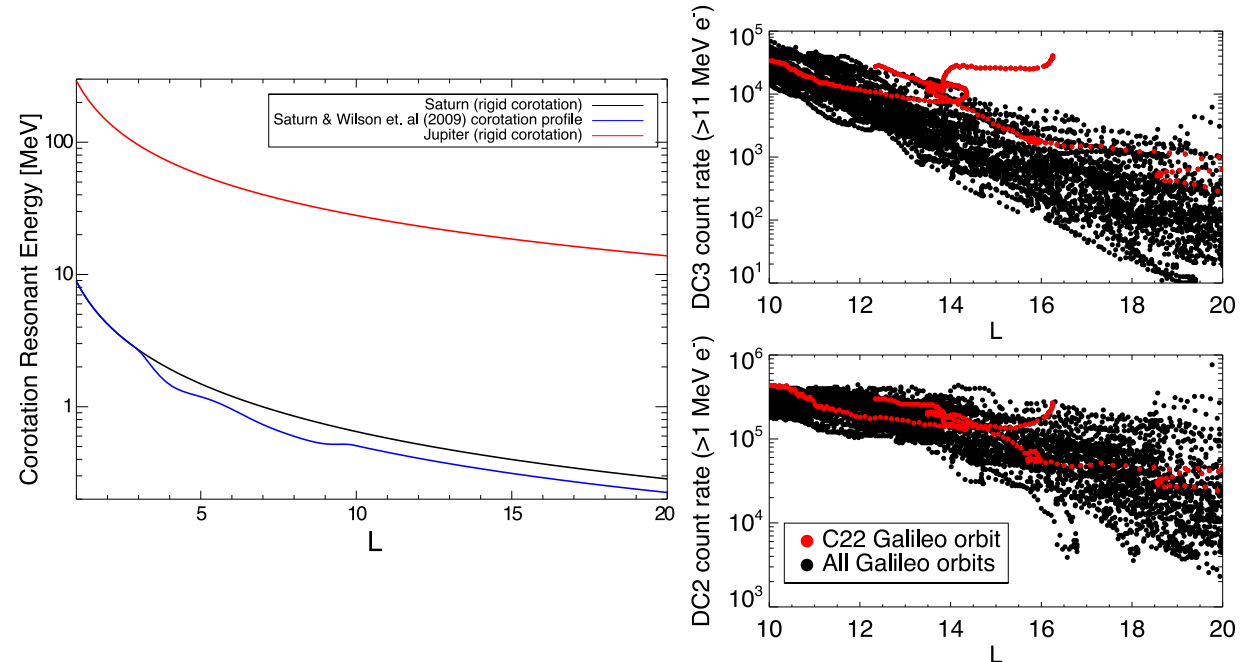

Figure 4. (Left) Electron energies as a function of L-shell, where electron drifts cancel-out with corotation (resonant energies), at Jupiter and Saturn. For Saturn, the blue curve shows these energies for a more realistic profile for corotation based on Wilson et al. [2009]. (Right) Galileo EPD measurements of $>1 \mathrm{MeV}$ and $>11$ $\mathrm{MeV}$ electrons as a function of $L$. The unusual $\mathrm{C} 22$ orbit shows a strong transient only at $>11 \mathrm{MeV}$, close to the resonant energy range for $\mathrm{L} \approx 15$. From Roussos et al. [2018d].

sidered to explain the bursts of synchrotron emissions seen at the time (e.g. de Pater et al. [1995]; Bolton and Thorne [1995]; Brecht et al. [2001]). SL9-type impacts are not frequent, but still not rare events, given Jupiter's strong gravity [Santos-Costa et al., 2011]. Changes in the solar EUV flux input to the jovian atmosphere correlate with 3-5 days delayed changes in the electron belts [Tsuchiya et al., 2011], which strongly supports the theoretical link between magnetospheric diffusion and variable thermospheric winds (Sec. 2.1.3).

$\mathrm{MeV}$ electron fluxes measured in-situ scatter over one order of magnitude for any given L-shell in the electron belt. More extreme transients, lasting for several days each (Galileo's orbits E06, C22), revealed $\mathrm{MeV}$ electron intensities rising above the average by factors of several hundred [Russell et al., 2001; Jun et al., 2005; Sorensen et al., 2005]. These enhancements were captured only in measurements of ultra-relativistic electrons ( $>11 \mathrm{MeV})$, hinting a similar acceleration mechanism as at Saturn, where electrons in drift-resonance with corotation are preferentially transported due to variable, convective flows (Fig. 4).

Such flows may be relevant to a dawn-dusk convective electric field in Jupiter's inner magnetosphere [Barbosa and Kivelson, 1983; Ip and Goertz, 1983], the amplitude of which enhances during solar wind induced compressions of the system [Murakami et al., 2016]. When radial diffusion rates estimates take into consideration these dawn-dusk electric field variations, the 0.7 or 2.7 years time lag in the response of the inner electron belts to long-term solar wind changes, can be replicated [?]. A similar electric field but with reversed pointing, controlled by diurnal atmospheric winds, is believed to modulate dawn-dusk asymmetries in the synchrotron part of the belt [Kita et al., 2015].

At lower energies, $<1 \mathrm{MeV}$, the intensity scatter between orbits is similarly large and mostly attributed to interchange injections [Mauk et al., 1999; Clark et al., 2016]. 


\section{Summary and conclusions}

Jupiter's and Saturn's radiation belts are strongly coupled to the characteristics of their host planet. This coupling highlights processes that are unresolved at Earth but may be critical for extraterrestrial radiation belts. Comparative studies between Earth, Jupiter and Saturn are therefore necessary for forming a more universal picture of how radiation belts work. Thanks to the nearly two-decade long datasets from Galileo, Juno and Cassini, such a comparison is now possible.

For instance, a seemingly unimportant detail such as the northward orientation of the jovian and saturnian magnetic fields, which controls the direction of energetic particle magnetic drifts, factors in to explain how very weak convective flows induced by the solar wind have a dominant control over electron transport and acceleration. So, even though Jupiter's and Saturn's magnetospheres are termed as "corotation-dominated", their $\mathrm{MeV}$ electron belt variability may be solar wind dominated. In addition, the perfect alignment of the magnetic and rotational axes at Saturn amplifies the role of energetic particle losses to moons and rings, explaining the much lower particle fluxes of the kronian belts compared to those of Jupiter, even though the two magnetospheres have many qualitative similarities. The study of the belt's seed populations also turns out to be an important one. Besides large scale dipolarization injections on the nightside magnetosphere, energetic particles accelerated near instantly to $\mathrm{MeV}$ energies at the aurora may also become trapped and a subsequent source of the radiation belts.

At present, the Juno mission is operating at Jupiter, collecting high quality, continuous measurements of the planet's magnetosphere and radiation belts at high latitudes and close to planet, regions that have not been covered by the Galileo orbiter. The 13-year Cassini dataset, and in particular the capabilities it offers for multi-instrument radiation belt studies, are gradually getting more exploited. The future Jupiter Icy Moons Explorer (JUICE) and Europa Clipper missions [Grasset et al., 2013; Pappalardo et al., 2017], while focusing primarily on the jovian moons Ganymede and Europa, respectively, will carry particles and fields instrumentation, including the first ever ENA imagers to the system, that will further enhance our understanding of the planet's radiation belts. The impact of the expected new measurements and investigations would significantly benefit planning of potential future missions to the magnetospheres of Uranus and Neptune and research on exoplanets which may host radiation belts.

\section{References}

Allen, R. C., D. G. Mitchell, C. P. Paranicas, D. C. Hamilton, G. Clark, A. M. Rymer, S. K. Vines, E. C. Roelof, S. M. Krimigis, and J. Vandegriff (2018), Internal Versus External Sources of Plasma at Saturn: Overview From Magnetospheric Imaging Investigation/Charge-Energy-Mass Spectrometer Data, Journal of Geophysical Research (Space Physics), 123, 4712-4727, doi:10.1029/2018JA025262.

Andriopoulou, M., E. Roussos, N. Krupp, C. Paranicas, M. Thomsen, S. Krimigis, M. K. Dougherty, and K.-H. Glassmeier (2012), A noon-to-midnight electric field and nightside dynamics in Saturn's inner magnetosphere, using microsignature observations, Icarus, 220, 503-513, doi:10.1016/j.icarus.2012.05.010.

Andriopoulou, M., E. Roussos, N. Krupp, C. Paranicas, M. Thomsen, S. Krimigis, M. Dougherty, and K.-H. Glassmeier (2013), Spatial and temporal dependence of the convective electric field in saturn's inner magnetosphere, Icarus, doi:http://dx.doi.org/10. 1016/j.icarus.2013.10.028.

Armstrong, T. P., S. Taherion, J. Manweiler, S. Krimigis, C. Paranicas, D. Mitchell, and N. Krupp (2009), Energetic ions trapped in Saturn's inner magnetosphere, Planet. Space Sci., 57, 1723-1731, doi:10.1016/j.pss.2009.03.008.

Badman, S. V., and S. W. H. Cowley (2007), Significance of Dungey-cycle flows in Jupiter's and Saturn's magnetospheres, and their identification on closed equatorial field lines, Ann. 
Geophys., 25, 941-951, doi:10.5194/angeo-25-941-2007.

Bagenal, F., R. J. Wilson, S. Siler, W. R. Paterson, and W. S. Kurth (2016), Survey of Galileo plasma observations in Jupiter's plasma sheet, J. Geophys. Res. (Planets), 121, 871-894, doi:10.1002/2016JE005009.

Barbosa, D. D., and M. G. Kivelson (1983), Dawn-dusk electric field asymmetry of the Io plasma torus, Geophys. Res. Lett., 10, 210-213, doi:10.1029/GL010i003p00210.

Becker, H. N., D. Santos-Costa, J. L. Jørgensen, T. Denver, A. Adriani, A. Mura, J. E. P. Connerney, S. J. Bolton, S. M. Levin, R. M. Thorne, J. W. Alexander, V. Adumitroaie, E. A. Manor-Chapman, I. J. Daubar, C. Lee, M. Benn, J. Sushkova, A. Cicchetti, and R. Noschese (2017), Observations of MeV electrons in Jupiter's innermost radiation belts and polar regions by the Juno radiation monitoring investigation: Perijoves 1 and 3, Geophys. Res. Lett., 44, 4481-4488, doi:10.1002/2017GL073091.

Birmingham, T. J. (1982), Charged particle motions in the distended magnetospheres of Jupiter and Saturn, Journal of Geophysical Research, 87, 7421-7430, doi:10.1029/ JA087iA09p07421.

Blanc, M., D. J. Andrews, A. J. Coates, D. C. Hamilton, C. M. Jackman, X. Jia, A. Kotova, M. Morooka, H. T. Smith, and J. H. Westlake (2015), Saturn Plasma Sources and Associated Transport Processes, Space Sci. Rev., 192, 237-283, doi:10.1007/s11214-015-0172-9.

Bolton, S. J., and R. M. Thorne (1995), Assessment of mechanisms for Jovian synchrotron variability associated with comet SL-9, Geophys. Res. Lett., 22, 1813-1816, doi:10.1029/ 95GL01515.

Bolton, S. J., R. M. Thorne, D. A. Gurnett, W. S. Kurth, and D. J. Williams (1997), Enhanced whistler-mode emissions: Signatures of interchange motion in the Io torus, Geophys. Res. Lett., 24, 2123, doi:10.1029/97GL02020.

Brecht, S. H., I. de Pater, D. J. Larson, and M. E. Pesses (2001), Modification of the Jovian Radiation Belts by Shoemaker-Levy 9: An Explanation of the Data, Icarus, 151, 25-38, doi:10.1006/icar.2001.6601.

Brice, N., and T. R. Mcdonough (1973), Jupiter's radiation belts, Icarus, 18, 206-219, doi: 10.1016/0019-1035(73)90204-2.

Buratti, B., et al. (2018), First results from cassini's five fabulous flybys of saturn's ring moons, Science.

Carbary, J. F., S. M. Krimigis, and W.-H. Ip (1983), Energetic particle microsignatures of Saturn's satellites, Journal Geophys. Res., 88, 8947-8958, doi:10.1029/ JA088iA11p08947.

Carbary, J. F., D. G. Mitchell, N. Krupp, and S. M. Krimigis (2009), L shell distribution of energetic electrons at Saturn, J. Geophys. Res. (Space Physics), 114, A09210, doi:10.1029/ 2009JA014341.

Cassidy, T. A., and R. E. Johnson (2010), Collisional spreading of Enceladus' neutral cloud, Icarus, 209, 696-703, doi:10.1016/j.icarus.2010.04.010.

Chen, Y., and T. W. Hill (2008), Statistical analysis of injection/dispersion events in Saturn's inner magnetosphere, J. Geophys. Res. (Space Physics), 113, 7215, doi:10.1029/ 2008JA013166.

Christon, S. P., D. C. Hamilton, R. D. Difabio, D. G. Mitchell, S. M. Krimigis, and D. S. Jontof-Hutter (2013), Saturn suprathermal $\mathrm{O}_{2}{ }^{+}$and mass- $-28^{+}$molecular ions: Long-term seasonal and solar variation, J. Geophys. Res., 118, 3446-3463, doi:10.1002/jgra.50383.

Clark, G., C. Paranicas, D. Santos-Costa, S. Livi, N. Krupp, D. G. Mitchell, E. Roussos, and W.-L. Tseng (2014), Evolution of electron pitch angle distributions across Saturn's middle magnetospheric region from MIMI/LEMMS, Planet. Space Sci., 104, 18-28, doi:10.1016/ j.pss.2014.07.004.

Clark, G., B. H. Mauk, C. Paranicas, P. Kollmann, and H. T. Smith (2016), Charge states of energetic oxygen and sulfur ions in Jupiter's magnetosphere, Journal of Geophysical Research (Space Physics), 121, 2264-2273, doi:10.1002/2015JA022257.

Clark, G., B. H. Mauk, C. Paranicas, D. Haggerty, P. Kollmann, A. Rymer, L. Brown, S. Jaskulek, C. Schlemm, C. Kim, J. Peachey, D. LaVallee, F. Allegrini, F. Bagenal, 
S. Bolton, J. Connerney, R. W. Ebert, G. Hospodarsky, S. Levin, W. S. Kurth, D. J. McComas, D. G. Mitchell, D. Ranquist, and P. Valek (2017), Observation and interpretation of energetic ion conics in Jupiter's polar magnetosphere, Geophys. Res. Lett., 44, 4419-4425, doi:10.1002/2016GL072325.

Clark, G., C. Tao, B. H. Mauk, J. Nichols, J. Saur, E. J. Bunce, F. Allegrini, R. Gladstone, F. Bagenal, S. Bolton, B. Bonfond, J. Connerney, R. W. Ebert, D. J. Gershman, D. Haggerty, T. Kimura, P. Kollmann, S. Kotsiaros, W. S. Kurth, S. Levin, D. J. McComas, G. Murakami, C. Paranicas, A. Rymer, and P. Valek (2018), Precipitating electron energy flux and characteristic energies in jupiter's main auroral region as measured by juno/jedi, J. Geophys. Res.: Space Physics, O(0), doi:10.1029/2018JA025639.

Cohen, C. M. S., E. C. Stone, and R. S. Selesnick (2001), Energetic ion observations in the middle Jovian magnetosphere, J. Geophys. Res., 106, 29,871-29,882, doi:10.1029/ 2001JA000008.

Connerney, J. E. P., S. Kotsiaros, R. J. Oliversen, J. R. Espley, J. L. Joergensen, P. S. Joergensen, J. M. G. Merayo, M. Herceg, J. Bloxham, K. M. Moore, S. J. Bolton, and S. M. Levin (2018), A New Model of Jupiter's Magnetic Field From Juno's First Nine Orbits, Geophys. Res. Lett., 45, 2590-2596, doi:10.1002/2018GL077312.

Cooper, J. F. (1983), Nuclear cascades in Saturn's rings - Cosmic ray albedo neutron decay and origins of trapped protons in the inner magnetosphere, Journal Geophys. Res., 88, 3945-3954, doi:10.1029/JA088iA05p03945.

Cooper, J. F., and S. J. Sturner (2018), Energetic radiation from galactic cosmic ray interactions with saturn's main rings, Journal of Geophysical Research: Space Physics, 123, doi:10.1029/2018JA025583.

de Pater, I., and C. K. Goertz (1994), Radial diffusion models of energetic electrons and jupiter's synchrotron radiation: 2. time variability, J. Geophys. Res. (Space Physics), 99(A2), 2271-2287, doi:10.1029/93JA02097.

de Pater, I., C. Heiles, M. Wong, R. J. Maddalena, M. K. Bird, O. Funke, J. Neidhoefer, R. M. Price, M. Kesteven, M. Calabretta, M. J. Klein, S. Gulkis, S. J. Bolton, R. S. Foster, S. Sukumar, R. G. Strom, R. S. Lepoole, T. Spoelstra, M. Robison, R. W. Hunstead, D. Campbell-Wilson, T. Ye, G. Dulk, Y. Leblanc, P. Galopeau, E. Gerard, and A. Lecacheux (1995), Outburst of Jupiter's Synchrotron Radiation After the Impact of Comet Shoemaker-Levy 9, Science, 268, 1879-1883, doi:10.1126/science.11536723.

de Soria-Santacruz, M., H. B. Garrett, R. W. Evans, I. Jun, W. Kim, C. Paranicas, and A. Drozdov (2016), An empirical model of the high-energy electron environment at Jupiter, Journal of Geophysical Research (Space Physics), 121, 9732-9743, doi:10.1002/ 2016JA023059.

de Soria-Santacruz, M., Y. Y. Shprits, A. Drozdov, J. D. Menietti, H. B. Garrett, H. Zhu, A. C. Kellerman, and R. B. Horne (2017), Interactions between energetic electrons and realistic whistler mode waves in the Jovian magnetosphere, Journal of Geophysical Research (Space Physics), 122, 5355-5364, doi:10.1002/2017JA023975.

Delamere, P. A., and F. Bagenal (2003), Modeling variability of plasma conditions in the io torus, Journal of Geophysical Research: Space Physics, 108(A7), doi:10.1029/ 2002JA009706.

Delamere, P. A., R. J. Wilson, S. Eriksson, and F. Bagenal (2013), Magnetic signatures of Kelvin-Helmholtz vortices on Saturn's magnetopause: Global survey, J. Geophys. Res. (Space Physics), 118, 393-404, doi:10.1029/2012JA018197.

Dialynas, K., S. M. Krimigis, D. G. Mitchell, D. C. Hamilton, N. Krupp, and P. C. Brandt (2009), Energetic ion spectral characteristics in the Saturnian magnetosphere using Cassini/MIMI measurements, J. Geophys. Res. (Space Physics), 114, A01212, doi: 10.1029/2008JA013761.

DiFabio, R. D., D. C. Hamilton, S. M. Krimigis, and D. G. Mitchell (2011), Long term time variations of the suprathermal ions in Saturn's magnetosphere, Geophys. Res. Lett., 38, L18103, doi:10.1029/2011GL048841. 
Dougherty, M. K., H. Cao, K. K. Khurana, G. J. Hunt, G. Provan, S. Kellock, M. E. Burton, T. A. Burk, E. J. Bunce, S. W. H. Cowley, M. G. Kivelson, C. T. Russell, and D. J. Southwood (2018), Saturn's magnetic field revealed by the cassini grand finale, Science, 362(6410), doi:10.1126/science.aat5434.

Drake, F. D., and S. Hvatum (1959), Non-thermal microwave radiation from Jupiter., AJ, 64, 329-330, doi:10.1086/108047.

Dumont, M., D. Grodent, A. Radioti, B. Bonfond, and J.-C. Gérard (2014), Jupiter's equatorward auroral features: Possible signatures of magnetospheric injections, Journal of Geophysical Research (Space Physics), 119, 10, doi:10.1002/2014JA020527.

Elrod, M. K., W.-L. Tseng, A. K. Woodson, and R. E. Johnson (2014), Seasonal and radial trends in Saturn's thermal plasma between the main rings and Enceladus, Icarus, 242, 130-137, doi:10.1016/j.icarus.2014.07.020.

Fischer, H. M., E. Pehlke, G. Wibberenz, L. J. Lanzerotti, and J. D. Mihalov (1996), Highenergy charged particles in the innermost jovian magnetosphere, Science, 272(5263), 856858, doi:10.1126/science.272.5263.856.

Fujiwara, K. (1976), Coulomb-Born Calculation of Charge Transfer Cross Sections of Highly-Ionized Atoms, Journal of the Physical Society of Japan, 41, 1350, doi:10.1143/ JPSJ.41.1350.

Galopeau, P. H. M., and E. Gérard (2001), Variations of Jupiter's synchrotron radiation: a link with solar activity?, Planet. Space Sci., 49, 1379-1391, doi:10.1016/S0032-0633(01) 00066-6.

Garrard, T. L., N. Gehrels, and E. C. Stone (1992), The galileo heavy element monitor, Space Science Reviews, 60(1), 305-315, doi:10.1007/BF00216859.

Garrett, H. B., L. M. Martinez-Sierra, and I. Jun (2015), Updating the Jovian Proton Radiation Environment - 2015, JPL Publication 15-9, https://trs.jpl.nasa.gov/handle/2014/45463.

Glass, G., M. Jain, M. L. Evans, J. C. Hiebert, L. C. Northcliffe, B. E. Bonner, J. E. Simmons, C. Bjork, P. Riley, and C. Cassapakis (1977), Neutron spectra at Odeg from protonproton collisions between 647 and $805 \mathrm{MeV}$, Phys. Rev. D, 15, 36-46, doi:10.1103/ PhysRevD.15.36.

Glauert, S. A., and R. B. Horne (2005), Calculation of pitch angle and energy diffusion coefficients with the padie code, Journal of Geophysical Research: Space Physics, 110(A4), doi:10.1029/2004JA010851.

Glauert, S. A., R. B. Horne, and N. P. Meredith (2014), Three-dimensional electron radiation belt simulations using the BAS Radiation Belt Model with new diffusion models for chorus, plasmaspheric hiss, and lightning-generated whistlers, Journal of Geophysical Research (Space Physics), 119, 268-289, doi:10.1002/2013JA019281.

Grasset, O., M. K. Dougherty, A. Coustenis, E. J. Bunce, C. Erd, D. Titov, M. Blanc, A. Coates, P. Drossart, L. N. Fletcher, H. Hussmann, R. Jaumann, N. Krupp, J. P. Lebreton, O. Prieto-Ballesteros, P. Tortora, F. Tosi, and T. Van Hoolst (2013), JUpiter ICy moons Explorer (JUICE): An ESA mission to orbit Ganymede and to characterise the Jupiter system, Planetary and Space Science, 78, 1-21, doi:10.1016/j.pss.2012.12.002.

Grießmeier, J.-M., P. Zarka, and J. N. Girard (2011), Observation of planetary radio emissions using large arrays, Radio Science, 46(5), doi:10.1029/2011RS004752.

Hedman, M. M., J. A. Burns, M. S. Tiscareno, C. C. Porco, G. H. Jones, E. Roussos, N. Krupp, C. Paranicas, and S. Kempf (2007), The Source of Saturn's G Ring, Science, 317, 653-, doi:10.1126/science.1143964.

Hess, W. N., H. W. Patterson, R. Wallace, and E. L. Chupp (1959), Cosmic-Ray Neutron Energy Spectrum, Phys. Rev., 116, 445-457, doi:10.1103/PhysRev.116.445.

Hood, L. L. (1983), Radial diffusion in Saturn's radiation belts - A modeling analysis assuming satellite and ring E absorption, Journal Geophys. Res., 88, 808-818, doi: 10.1029/JA088iA02p00808.

Horne, R. B., and R. M. Thorne (2003), Relativistic electron acceleration and precipitation during resonant interactions with whistler-mode chorus, Geophys. Res. Lett., 30, 1527, 
doi:10.1029/2003GL016973.

Huddleston, D. E., R. J. Strangeway, J. Warnecke, C. T. Russell, and M. G. Kivelson (1998), Ion cyclotron waves in the Io torus: Wave dispersion, free energy analysis, and $\mathrm{SO} 2$ source rate estimates, J. Geophys. Res., 103, 19,887-19,900, doi:10.1029/97JE03557.

Ip, W.-H., and C. K. Goertz (1983), An interpretation of the dawn-dusk asymmetry of UV emission from the Io plasma torus, Nature, 302, 232, doi:10.1038/302232a0.

Johnson, R. E., J. G. Luhmann, R. L. Tokar, M. Bouhram, J. J. Berthelier, E. C. Sittler, J. F. Cooper, T. W. Hill, H. T. Smith, M. Michael, M. Liu, F. J. Crary, and D. T. Young (2006), Production, ionization and redistribution of $\mathrm{O}_{2}$ in Saturn's ring atmosphere, Icarus, 180, 393-402, doi:10.1016/j.icarus.2005.08.021.

Johnson, R. E., O. J. Tucker, M. Michael, E. C. Sittler, H. T. Smith, D. T. Young, and J. H. Waite (2010), Mass Loss Processes in Titan's Upper Atmosphere, p. 373, doi:10.1007/ 978-1-4020-9215-2-15.

Joy, S. P., M. G. Kivelson, R. J. Walker, K. K. Khurana, C. T. Russell, and T. Ogino (2002), Probabilistic models of the Jovian magnetopause and bow shock locations, Journal of Geophysical Research (Space Physics), 107, 1309, doi:10.1029/2001JA009146.

Jun, I., H. B. Garrett, R. Swimm, R. W. Evans, and G. Clough (2005), Statistics of the variations of the high-energy electron population between 7 and 28 jovian radii as measured by the Galileo spacecraft, Icarus, 178, 386-394, doi:10.1016/j.icarus.2005.01.022.

Jurac, S., R. E. Johnson, J. D. Richardson, and C. Paranicas (2001), Satellite sputtering in Saturn's magnetosphere, Planet. Space Sci., 49, 319-326.

Kanani, S. J., C. S. Arridge, G. H. Jones, A. N. Fazakerley, H. J. McAndrews, N. Sergis, S. M. Krimigis, M. K. Dougherty, A. J. Coates, D. T. Young, K. C. Hansen, and N. Krupp (2010), A new form of Saturn's magnetopause using a dynamic pressure balance model, based on in situ, multi-instrument Cassini measurements, J. Geophys. Res. (Space Physics), 115, A06207, doi:10.1029/2009JA014262.

Kennel, C. F., and H. E. Petschek (1966), Limit on Stably Trapped Particle Fluxes, J. Geophys. Res., 71, 1, doi:10.1029/JZ071i001p00001.

Khurana, K. K., and M. G. Kivelson (1989), Ultralow frequency MHD waves in Jupiter's middle magnetosphere, J. Geophys. Res., 94, 5255-5263.

Kim, T. K., and et al. (2018), Properties of Jupiter's Plasma Sheet Ions Observed by Juno JADE Including Estimates of in-situ Abundance for O+ and $\mathrm{S} 2+$, J. Geophys. Res., submitted.

Kita, H., H. Misawa, A. Bhardwaj, F. Tsuchiya, T. Sakanoi, Y. Kasaba, C. Tao, Y. Miyoshi, and A. Morioka (2015), Relation between the short-term variation of the Jovian radiation belt and thermosphere derived from radio and infrared observations, Journal of Geophysical Research (Space Physics), 120, 6614-6623, doi:10.1002/2015JA021374.

Kivelson, M. G. (2006), Does Enceladus Govern Magnetospheric Dynamics at Saturn?, Science, 311, 1391-1392, doi:10.1126/science.1124494.

Kivelson, M. G. (2007), Planetary Magnetospheres, pp. 469-492, Springer Berlin Heidelberg, Berlin, Heidelberg.

Kollmann, P., E. Roussos, C. Paranicas, N. Krupp, C. M. Jackman, E. Kirsch, and K.H. Glassmeier (2011), Energetic particle phase space densities at Saturn: Cassini observations and interpretations, J. Geophys. Res. (Space Physics), 116, A05,222, doi: 10.1029/2010JA016221.

Kollmann, P., E. Roussos, C. Paranicas, N. Krupp, and D. K. Haggerty (2013), Processes forming and sustaining Saturn's proton radiation belts, Icarus, 222, 323-341, doi:10.1016/ j.icarus.2012.10.033.

Kollmann, P., E. Roussos, A. Kotova, J. F. Cooper, D. G. Mitchell, N. Krupp, and C. Paranicas (2015), Mev proton flux predictions near saturn's d ring, J. Geophys. Res. (Space Physics), 120(10), 8586-8602, doi:10.1002/2015JA021621.

Kollmann, P., C. Paranicas, G. Clark, E. Roussos, A. Lagg, and N. Krupp (2016), The vertical thickness of Jupiter's Europa gas torus from charged particle measurements, Geophys. Res. Lett., 43, 9425-9433, doi:10.1002/2016GL070326. 
Kollmann, P., E. Roussos, A. Kotova, C. Paranicas, and N. Krupp (2017a), The evolution of Saturn's radiation belts modulated by changes in radial diffusion, Nature Astronomy, doi: 10.1038/s41550-017-0287-x.

Kollmann, P., C. Paranicas, G. Clark, B. H. Mauk, D. K. Haggerty, A. M. Rymer, D. SantosCosta, J. E. P. Connerney, F. Allegrini, P. Valek, W. S. Kurth, G. R. Gladstone, S. Levin, and S. Bolton (2017b), A heavy ion and proton radiation belt inside of jupiter's rings, Geophys. Res. Lett., 44(11), 5259-5268, doi:10.1002/2017GL073730.

Kollmann, P., E. Roussos, C. P. Paranicas, E. E. Woodfield, B. H. Mauk, G. Clark, D. C. Smith, and J. Vandegriff (2018a), Electron acceleration to mev energies at jupiter and saturn, Journal of Geophysical Research (Space Physics), doi:10.1029/2018JA025665.

Kollmann, P., E. Roussos, A. Kotova, L. Regoli, D. G. Mitchell, J. Carbary, G. Clark, N. Krupp, and C. Paranicas (2018b), Saturn's innermost radiation belt throughout and inward of the D-ring, Geophys. Res. Lett., 45, doi:10.1029/2018GL077954.

Kotova, A. (2016), Energetic particle tracking techniques and its application to the magnetosphere of Saturn, Theses, Uninersite Toulouse III - Paul Sabatier.

Kotova, A., E. Roussos, P. Kollmann, N. Krupp, and I. Dandouras (2018), Galactic Cosmic Rays access to the magnetosphere of Saturn, J. Geophys. Res., under revision.

Krimigis, S. M., D. G. Mitchell, D. C. Hamilton, S. Livi, J. Dandouras, S. Jaskulek, T. P. Armstrong, J. D. Boldt, A. F. Cheng, G. Gloeckler, J. R. Hayes, K. C. Hsieh, W.-H. Ip, E. P. Keath, E. Kirsch, N. Krupp, L. J. Lanzerotti, R. Lundgren, B. H. Mauk, R. W. McEntire, E. C. Roelof, C. E. Schlemm, B. E. Tossman, B. Wilken, and D. J. Williams (2004), Magnetosphere Imaging Instrument (MIMI) on the Cassini Mission to Saturn/Titan, Space Science Reviews, 114, 233-329, doi:10.1007/s11214-004-1410-8.

Krimigis, S. M., D. G. Mitchell, D. C. Hamilton, N. Krupp, S. Livi, E. C. Roelof, J. Dandouras, T. P. Armstrong, B. H. Mauk, C. Paranicas, P. C. Brandt, S. Bolton, A. F. Cheng, T. Choo, G. Gloeckler, J. Hayes, K. C. Hsieh, W.-H. Ip, S. Jaskulek, E. P. Keath, E. Kirsch, M. Kusterer, A. Lagg, L. J. Lanzerotti, D. LaVallee, J. Manweiler, R. W. McEntire, W. Rasmuss, J. Saur, F. S. Turner, D. J. Williams, and J. Woch (2005), Dynamics of saturn's magnetosphere from mimi during cassini's orbital insertion, Science, 307(5713), 1270-1273, doi:10.1126/science.1105978.

Kronberg, E. A., K.-H. Glassmeier, J. Woch, N. Krupp, A. Lagg, and M. K. Dougherty (2007), A possible intrinsic mechanism for the quasi-periodic dynamics of the Jovian magnetosphere, Journal of Geophysical Research (Space Physics), 112, A05203, doi: 10.1029/2006JA011994.

Krupp, N., E. Roussos, P. Kollmann, C. Paranicas, D. G. Mitchell, S. M. Krimigis, A. Rymer, C. S. Arridge, T. P. Armstrong, and K. K. Khurana (2012), The Cassini Enceladus encounters 2005-2010 in the view of energetic electron measurements, Icarus, 218, doi: 10.1016/j.icarus.2011.12.018.

Krupp, N., E. Roussos, C. Paranicas, A. Sicard, G. Hospodarsky, and Y. Shprits (2016), chap. Energetic Particles and Waves in the Outer Planet Radiation Belts, Oxford University Press, Oxford.

Krupp, N., E. Roussos, P. Kollmann, D. G. Mitchell, C. P. Paranicas, S. M. Krimigis, D. C. Hamilton, M. Hedman, and M. K. Dougherty (2018), Energetic neutral and charged particle measurements in the inner Saturnian magnetosphere during the Grand Finale orbits of Cassini 2016/2017, Geophys. Res. Lett., doi:10.1029/2018GL078096.

Lagg, A., N. Krupp, J. Woch, and D. J. Williams (2003), In-situ observations of a neutral gas torus at Europa, Geophys. Res. Lett., 30(11), 1556, doi:10.1029/2003GL017214.

Li, X., R. Selesnick, Q. Schiller, K. Zhang, H. Zhao, D. N. Baker, and M. A. Temerin (2017), Measurement of electrons from albedo neutron decay and neutron density in near-Earth space, Nature, 552, 382-385, doi:10.1038/nature24642.

Liu, X., and T. W. Hill (2012), Effects of finite plasma pressure on centrifugally driven convection in Saturn's inner magnetosphere, J. Geophys. Res., 117, A07216, doi:10.1029/ 2012JA017827. 
Lorenzato, L., A. Sicard, and S. Bourdarie (2012), A physical model for electron radiation belts of Saturn, J. Geophys. Res. (Space Physics), 117, A08214, doi:10.1029/ 2012JA017560.

Louarn, P., C. P. Paranicas, and W. S. Kurth (2014), Global magnetodisk disturbances and energetic particle injections at Jupiter, Journal of Geophysical Research (Space Physics), 119, 4495-4511, doi:10.1002/2014JA019846.

Mauk, B. H. (2014), Comparative investigation of the energetic ion spectra comprising the magnetospheric ring currents of the solar system, J. Geophys. Res., 119, 9729-9746, doi: 10.1002/2014JA020392.

Mauk, B. H., and N. J. Fox (2010), Electron radiation belts of the solar system, J. Geophys. Res. (Space Physics), 115, 12,220, doi:10.1029/2010JA015660.

Mauk, B. H., D. J. Williams, R. W. McEntire, K. K. Khurana, and J. G. Roederer (1999), Storm-like dynamics of jupiter's inner and middle magnetosphere, J. Geophys. Res. (Space Physics), 104(A10), 22,759-22,778, doi:10.1029/1999JA900097.

Mauk, B. H., D. G. Mitchell, R. W. McEntire, C. P. Paranicas, E. C. Roelof, D. J. Williams, S. M. Krimigis, and A. Lagg (2004), Energetic ion characteristics and neutral gas interactions in jupiter's magnetosphere, J. Geophys. Res. (Space Physics), 109(A9), doi: 10.1029/2003JA010270, a09S12.

Mauk, B. H., N. J. Fox, S. G. Kanekal, R. L. Kessel, D. G. Sibeck, and A. Ukhorskiy (2013), Science objectives and rationale for the radiation belt storm probes mission, Space Science Reviews, 179(1), 3-27, doi:10.1007/s11214-012-9908-y.

Mauk, B. H., D. K. Haggerty, C. Paranicas, G. Clark, P. Kollmann, A. M. Rymer, J. M. Peachey, S. J. Bolton, S. M. Levin, A. Adriani, F. Allegrini, F. Bagenal, B. Bonfond, J. E. P. Connerney, R. W. Ebert, G. R. Gladstone, W. S. Kurth, D. J. McComas, D. Ranquist, and P. Valek (2018), Diverse Electron and Ion Acceleration Characteristics Observed Over Jupiter's Main Aurora, Geophys. Res. Lett., 45, 1277-1285, doi:10.1002/ 2017 GL076901.

McKibben, R. B., J. A. Simpson, and M. Zhang (1993), Impulsive bursts of relativistic electrons discovered during Ulysses' traversal of Jupiter's dusk-side magnetosphere, Plan. Space Sci., 41, 1041-1058, doi:10.1016/0032-0633(93)90108-E.

Meeks, Z., S. Simon, and S. Kabanovic (2016), A comprehensive analysis of ion cyclotron waves in the equatorial magnetosphere of Saturn, Planet. Space Sci., 129, 47-60, doi:10. 1016/j.pss.2016.06.003.

Menietti, J. D., T. F. Averkamp, J. B. Groene, R. B. Horne, Y. Y. Shprits, E. E. Woodfield, G. B. Hospodarsky, and D. A. Gurnett (2014), Survey analysis of chorus intensity at Saturn, J. Geophys. Res., 119, 8415-8425, doi:10.1002/2014JA020523.

Menietti, J. D., J. B. Groene, T. F. Averkamp, R. B. Horne, E. E. Woodfield, Y. Y. Shprits, M. Soria-Santacruz Pich, and D. A. Gurnett (2016), Survey of whistler mode chorus intensity at Jupiter, J. Geophys. Res., 121, 9758-9770, doi:10.1002/2016JA022969.

Milillo, A., C. Plainaki, E. De Angelis, V. Mangano, S. Massetti, A. Mura, S. Orsini, and R. Rispoli (2016), Analytical model of Europa's $\mathrm{O}_{2}$ exosphere, Planet. Space Sci., 130, 3-13, doi:10.1016/j.pss.2015.10.011.

Miyoshi, Y., H. Misawa, A. Morioka, T. Kondo, Y. Koyama, and J. Nakajima (1999), Observation of short-term variation of Jupiter's synchrotron radiation, Geophys. Res. Lett., 26, 9-12, doi:10.1029/1998GL900244.

Murakami, G., K. Yoshioka, A. Yamazaki, F. Tsuchiya, T. Kimura, C. Tao, H. Kita, M. Kagitani, T. Sakanoi, K. Uemizu, Y. Kasaba, I. Yoshikawa, and M. Fujimoto (2016), Response of jupiter's inner magnetosphere to the solar wind derived from extreme ultraviolet monitoring of the io plasma torus, Geophys. Res. Lett., 43(24), 12,308-12,316, doi: 10.1002/2016GL071675, 2016GL071675.

Nénon, Q., A. Sicard, and S. Bourdarie (2017), A new physical model of the electron radiation belts of Jupiter inside Europa's orbit, J. Geophys. Res. (Space Physics), 122, 51485167, doi:10.1002/2017JA023893. 
Nénon, Q., A. Sicard, P. Kollmann, H. B. Garrett, S. P. A. Sauer, and C. Paranicas (2018), A Physical Model of the Proton Radiation Belts of Jupiter inside Europa's Orbit, Journal of Geophysical Research (Space Physics), 123, 3512-3532, doi:10.1029/2018JA025216.

Nenon, Q., A. Sicard, and P. Caron (2018), The rings of jupiter as seen by the electron and proton radiation belt model salammbo, Geophys. Res. Lett., doi:10.1029/2018g1080157.

Palmaerts, B., E. Roussos, N. Krupp, W. S. Kurth, D. G. Mitchell, and J. N. Yates (2016), Statistical analysis and multi-instrument overview of the quasi-periodic 1-hour pulsations in Saturn's outer magnetosphere, Icarus, 271, 1-18, doi:10.1016/j.icarus.2016.01.025.

Pappalardo, R. T., D. A. Senske, H. Korth, D. Blankenship, D. Blaney, P. Christensen, S. Kempf, C. Raymond, K. Retherford, E. P. Turtle, J. H. Waite, J. Westlake, G. C. Collins, K. Hand, J. Lunine, M. McGrath, F. Nimmo, C. Paty, J. Soderblom, J. R. Spencer, C. Paranicas, S. Solomon, and Europa Science Team (2017), The Europa Multiple-Flyby Mission: Synergistic Science to Investigate Habitability, in Lunar and Planetary Science Conference, p. 2732.

Paranicas, C., W. R. Paterson, A. F. Cheng, B. H. Mauk, R. W. McEntire, L. A. Frank, and D. J. Williams (1999), Energetic particle observations near Ganymede, J. Geophys. Res., 104, 17,459-17,470, doi:10.1029/1999JA900199.

Paranicas, C., J. M. Ratliff, B. H. Mauk, C. Cohen, and R. E. Johnson (2002), The ion environment near Europa and its role in surface energetics, Geophys. Res. Lett., 29, 1074, doi:10.1029/2001GL014127.

Paranicas, C., D. G. Mitchell, S. M. Krimigis, D. C. Hamilton, E. Roussos, N. Krupp, G. H. Jones, R. E. Johnson, J. F. Cooper, and T. P. Armstrong (2008), Sources and losses of energetic protons in Saturn's magnetosphere, Icarus, 197, 519-525, doi:10.1016/j.icarus. 2008.05.011.

Paranicas, C., D. G. Mitchell, E. Roussos, P. Kollmann, N. Krupp, A. L. Müller, S. M. Krimigis, F. S. Turner, P. C. Brandt, A. M. Rymer, and R. E. Johnson (2010a), Transport of energetic electrons into Saturn's inner magnetosphere, J. Geophys. Res. (Space Physics), 115, 9214, doi:10.1029/2010JA015853.

Paranicas, C., D. G. Mitchell, S. M. Krimigis, J. F. Carbary, P. C. Brandt, F. S. Turner, E. Roussos, N. Krupp, M. G. Kivelson, K. K. Khurana, J. F. Cooper, T. P. Armstrong, and M. Burton (2010b), Asymmetries in Saturn's radiation belts, J. Geophys. Res. (Space Physics), 115(A14), A07216, doi:10.1029/2009JA014971.

Paranicas, C., M. F. Thomsen, N. Achilleos, M. Andriopoulou, S. V. Badman, G. Hospodarsky, C. M. Jackman, X. Jia, T. Kennelly, K. Khurana, P. Kollmann, N. Krupp, P. Louarn, E. Roussos, and N. Sergis (2016), Effects of radial motion on interchange injections at Saturn, Icarus, 264, 342-351, doi:10.1016/j.icarus.2015.10.002.

Paranicas, C., B. H. Mauk, D. K. Haggerty, G. Clark, P. Kollmann, A. M. Rymer, B. Bonfond, W. R. Dunn, R. W. Ebert, G. R. Gladstone, E. Roussos, N. Krupp, F. Bagenal, S. M. Levin, J. E. P. Connerney, and S. J. Bolton (2018), Intervals of Intense Energetic Electron Beams Over Jupiter's Poles, Journal of Geophysical Research (Space Physics), 123, 19891999, doi:10.1002/2017JA025106.

Pehlke, E. (2000), Teilchenpopulationen in der inneren jupitermagnetosphäre - untersuchung der epi-daten von der galileo-probe, Ph.D. thesis, University of Kiel.

Persoon, A. M., D. A. Gurnett, J. S. Leisner, W. S. Kurth, J. B. Groene, and J. B. Faden (2013), The plasma density distribution in the inner region of Saturn's magnetosphere, J. Geophys. Res., 118, 2970-2974, doi:10.1002/jgra.50182.

Radioti, A., J. Woch, E. A. Kronberg, N. Krupp, A. Lagg, K.-H. Glassmeier, and M. K. Dougherty (2007), Energetic ion composition during reconfiguration events in the Jovian magnetotail, Journal of Geophysical Research (Space Physics), 112, A06221, doi: 10.1029/2006JA012047.

Roederer, J. G. (1970), Dynamics of geomagnetically trapped radiation.

Roussos, E., G. H. Jones, N. Krupp, C. Paranicas, D. G. Mitchell, A. Lagg, J. Woch, U. Motschmann, S. M. Krimigis, and M. K. Dougherty (2007), Electron microdiffusion in the Saturnian radiation belts: Cassini MIMI/LEMMS observations of energetic 
electron absorption by the icy moons, J. Geophys. Res. (Space Physics), 112, 6214, doi: 10.1029/2006JA012027.

Roussos, E., N. Krupp, T. P. Armstrong, C. Paranicas, D. G. Mitchell, S. M. Krimigis, G. H. Jones, K. Dialynas, N. Sergis, and D. C. Hamilton (2008), Discovery of a transient radiation belt at Saturn, Geophys. Res. Lett., 35, 22,106, doi:10.1029/2008GL035767.

Roussos, E., N. Krupp, C. P. Paranicas, P. Kollmann, D. G. Mitchell, S. M. Krimigis, T. P. Armstrong, D. R. Went, M. K. Dougherty, and G. H. Jones (2011), Long- and shortterm variability of Saturn's ionic radiation belts, J. Geophys. Res. (Space Physics), 116, A02,217, doi:10.1029/2010JA015954.

Roussos, E., N. Krupp, C. Paranicas, J. F. Carbary, P. Kollmann, S. M. Krimigis, and D. G. Mitchell (2014), The variable extension of Saturn's electron radiation belts, Planetary Space Sci., 104, 3-17, doi:10.1016/j.pss.2014.03.021.

Roussos, E., N. Krupp, D. G. Mitchell, C. Paranicas, S. M. Krimigis, M. Andriopoulou, B. Palmaerts, W. S. Kurth, S. V. Badman, A. Masters, and M. K. Dougherty (2016), Quasi-periodic injections of relativistic electrons in Saturn's outer magnetosphere, Icarus, 263, 101-116, doi:10.1016/j.icarus.2015.04.017.

Roussos, E., P. Kollmann, N. Krupp, C. Paranicas, K. Dialynas, G. H. Jones, D. G. Mitchell, S. M. Krimigis, and J. F. Cooper (2018a), Sources, sinks and transport of energetic electrons near saturns main rings, Geophysical Research Letters, doi:10.1029/2018g1078097.

Roussos, E., P. Kollmann, N. Krupp, A. Kotova, L. Regoli, C. Paranicas, D. G. Mitchell, S. M. Krimigis, D. Hamilton, P. Brandt, J. Carbary, S. Christon, K. Dialynas, I. Dandouras, M. E. Hill, W. H. Ip, G. H. Jones, S. Livi, B. H. Mauk, B. Palmaerts, E. C. Roelof, A. Rymer, N. Sergis, and H. T. Smith (2018b), A radiation belt of energetic protons located between saturn and its rings, Science, 362(6410), doi:10.1126/science.aat1962.

Roussos, E., C. Jackman, M. Thomsen, W. Kurth, S. Badman, C. Paranicas, P. Kollmann, N. Krupp, R. Bučík, D. Mitchell, S. Krimigis, D. Hamilton, and A. Radioti (2018c), Solar energetic particles (sep) and galactic cosmic rays (gcr) as tracers of solar wind conditions near saturn: Event lists and applications, Icarus, 300, 47 - 71, doi:https://doi.org/10.1016/ j.icarus.2017.08.040.

Roussos, E., P. Kollmann, N. Krupp, C. Paranicas, K. Dialynas, N. Sergis, D. Mitchell, D. Hamilton, and S. Krimigis (2018d), Drift-resonant, relativistic electron acceleration at the outer planets: Insights from the response of saturn's radiation belts to magnetospheric storms, Icarus, 305, 160 - 173, doi:https://doi.org/10.1016/j.icarus.2018.01.016.

Russell, C. T., P. D. Fieseler, D. Bindshadler, Z. J. Yu, S. P. Joy, K. K. Khurana, and M. G. Kivelson (2001), Large scale changes in the highly energetic charged particles in the region of the Io torus, Advances in Space Research, 28, 1495-1500, doi:10.1016/ S0273-1177(01)00552-X.

Rymer, A. M., B. H. Mauk, T. W. Hill, C. Paranicas, D. G. Mitchell, A. J. Coates, and D. T. Young (2008), Electron circulation in Saturn's magnetosphere, J. Geophys. Res. (Space Physics), 113, 1201, doi:10.1029/2007JA012589.

Santos-Costa, D., and S. J. Bolton (2008), Discussing the processes constraining the Jovian synchrotron radio emission's features, Planet. Space Sci., 56, 326-345, doi:10.1016/j.pss. 2007.09.008.

Santos-Costa, D., and S. A. Bourdarie (2001), Modeling the inner Jovian electron radiation belt including non-equatorial particles, Planet. Space Sci., 49, 303-312, doi: 10.1016/S0032-0633(00)00151-3.

Santos-Costa, D., M. Blanc, S. Maurice, and S. J. Bolton (2003), Modeling the electron and proton radiation belts of saturn, Geophys. Res. Lett., 30(20), doi:10.1029/2003GL017972, 2059.

Santos-Costa, D., S. J. Bolton, R. M. Thorne, Y. Miyoshi, and S. M. Levin (2008), Investigating the origins of the Jovian decimetric emission's variability, Journal of Geophysical Research (Space Physics), 113, A01204, doi:10.1029/2007JA012396.

Santos-Costa, D., S. J. Bolton, R. J. Sault, R. M. Thorne, and S. M. Levin (2011), VLA observations at $6.2 \mathrm{~cm}$ of the response of Jupiter's electron belt to the July 2009 event, Jour- 
nal of Geophysical Research (Space Physics), 116, A12236, doi:10.1029/2011JA016921.

Sauer, H. H. (1980), On Saturnian cosmic ray cutoff rigidities, Geophys. Res. Lett., 7, $215-$ 217, doi:10.1029/GL007i003p00215.

Schulz, M., and L. J. Lanzerotti (1974), Particle Diffusion in the Radiation Belts, in Physics and Chemistry in Space 7, edited by J. G. Roederer, 1st ed., Springer Verlag, Heidelberg, Germany.

Seidelmann, P. K., and N. Divine (1977), Evaluation of Jupiter longitudes in System III/1965/, Geophys. Res. Lett., 4, 65-68, doi:10.1029/GL004i002p00065.

Selesnick, R. S., and C. M. S. Cohen (2009), Charge states of energetic ions in Jupiter's radiation belt inferred from absorption microsignatures of Io, Journal of Geophysical Research (Space Physics), 114, A01207, doi:10.1029/2008JA013722.

Selesnick, R. S., C. M. S. Cohen, and E. C. Stone (2001a), Mapping Jupiter's outer radiation belt, J. Geophys. Res., 106, 29,859-29,870, doi:10.1029/2001JA000061.

Selesnick, R. S., C. M. S. Cohen, and K. K. Khurana (2001b), Energetic ion dynamics in Jupiter's plasma sheet, J. Geophys. Res., 106, 18,895-18,906, doi:10.1029/2000JA000242.

Sergis, N., S. M. Krimigis, D. G. Mitchell, D. C. Hamilton, N. Krupp, B. M. Mauk, E. C. Roelof, and M. Dougherty (2007), Ring current at Saturn: Energetic particle pressure in Saturn's equatorial magnetosphere measured with Cassini/MIMI, Geophys. Res. Lett., 34, L09102, doi:10.1029/2006GL029223.

Shikaze, Y., S. Haino, K. Abe, H. Fuke, T. Hams, K. C. Kim, Y. Makida, S. Matsuda, J. W. Mitchell, A. A. Moiseev, J. Nishimura, M. Nozaki, S. Orito, J. F. Ormes, T. Sanuki, M. Sasaki, E. S. Seo, R. E. Streitmatter, J. Suzuki, K. Tanaka, T. Yamagami, A. Yamamoto, T. Yoshida, and K. Yoshimura (2007), Measurements of $0.220 \mathrm{GeV} / \mathrm{n}$ cosmicray proton and helium spectra from 1997 through 2002 with the BESS spectrometer, Astropart. Phys., 28, 154-167, doi:10.1016/j.astropartphys.2007.05.001.

Shprits, Y. Y., J. D. Menietti, X. Gu, K. C. Kim, and R. B. Horne (2012), Gyroresonant interactions between the radiation belt electrons and whistler mode chorus waves in the radiation environments of Earth, Jupiter, and Saturn: A comparative study, J. Geophys. Res. (Space Physics), 117, A11216, doi:10.1029/2012JA018031.

Shprits, Y. Y., J. D. Menietti, A. Y. Drozdov, R. B. Horne, E. E. Woodfield, J. B. Groene, M. de Soria-Santacruz, T. F. Averkamp, H. Garrett, C. Paranicas, and D. A. Gurnett (2018), Strong whistler mode waves observed in the vicinity of jupiter's moons, Nature Communications, 9(1), 3131, doi:10.1038/s41467-018-05431-X.

Sicard-Piet, A., S. Bourdarie, and N. Krupp (2011), Jose: A new jovian specification environment model, IEEE Transactions on Nuclear Science, 58(3), 923-931, doi:10.1109/ TNS.2010.2097276.

Smith, H. T., F. J. Crary, M. K. Dougherty, M. E. Perry, E. Roussos, S. Simon, R. L. Tokar, and R. Dotson (2018), Enceladus and Its Influence on Saturn's Magnetosphere, pp. 211234, University of Arizona Press.

Smith, H. T., D. G. Mitchell, R. E. Johnson, B. H. Mauk, and J. E. Smith (2019), Europa Neutral Torus Confirmation and Characterization Based on Observations and Modeling, ApJ, 871, 69, doi:10.3847/1538-4357/aaed38.

Smyth, W. H., and M. L. Marconi (2006), Europa's atmosphere, gas tori, and magnetospheric implications, Icarus, 181, 510-526, doi:10.1016/j.icarus.2005.10.019.

Sorensen, T. C., T. P. Armstrong, A. Gandhi Pavanasam, and S. Taherion (2005), Galileo energetic particle detector observations of the spatial distributions and energy spectra of $>1$ and $>11 \mathrm{MeV}$ electrons in the 10-40 $\mathrm{R}_{s}$ region of the jovian magnetosphere, Icarus, 178, 395-405, doi:10.1016/j.icarus.2005.07.006.

Southwood, D. J., and M. G. Kivelson (1987), Magnetospheric interchange instability, J. Geophys. Res., 92, 109-116, doi:10.1029/JA092iA01p00109.

Speiser, T. W. (1965), Particle Trajectories in Model Current Sheets, 1, Analytical Solutions, J. Geophys. Res., 70, 4219-4226, doi:10.1029/JZ070i017p04219.

Summers, D., R. Tang, and Y. Omura (2011), Effects of nonlinear wave growth on extreme radiation belt electron fluxes, Journal of Geophysical Research (Space Physics), 116, 
A10226, doi:10.1029/2011JA016602.

Taherion, S., T. P. Armstrong, and H. B. Garrett (2008), Ultrarelativistic electrons in jupiter's inner magnetosphere: First observation of angular distributions in the 2.5 to 6 rj region, The Astrophysical Journal Letters, 685(1), L79.

Teolis, B. D., G. H. Jones, P. F. Miles, R. L. Tokar, B. A. Magee, J. H. Waite, E. Roussos, D. T. Young, F. J. Crary, A. J. Coates, R. E. Johnson, W.-L. Tseng, and R. A. Baragiola (2010), Cassini Finds an Oxygen-Carbon Dioxide Atmosphere at Saturn's Icy Moon Rhea, Science, 330, 1813-, doi:10.1126/science.1198366.

Thomsen, M. F., and J. A. Van Allen (1980), Motion of trapped electrons and protons in Saturn's inner magnetosphere, Journal Geophys. Res., 85, 5831-5834, doi:10.1029/ JA085iA11p05831.

Thomsen, M. F., C. K. Goertz, and J. A. Van Allen (1977), On determining magnetospheric diffusion coefficients from the observed effects of Jupiter's satellite Io, J. Geophys. Res., 82, 5541-5550, doi:10.1029/JA082i035p05541.

Thomsen, M. F., E. Roussos, M. Andriopoulou, P. Kollmann, C. S. Arridge, C. P. Paranicas, D. A. Gurnett, R. L. Powell, R. L. Tokar, and D. T. Young (2012), Saturn's inner magnetospheric convection pattern: Further evidence, J. Geophys. Res. (Space Physics), 117, A09208, doi:10.1029/2011JA017482.

Thomsen, M. F., A. J. Coates, E. Roussos, R. J. Wilson, K. C. Hansen, and G. R. Lewis (2016), Suprathermal electron penetration into the inner magnetosphere of saturn, J. Geophys. Res. (Space Physics), 121(6), 5436-5448, doi:10.1002/2016JA022692, 2016JA022692.

Tomás, A. T., J. Woch, N. Krupp, A. Lagg, K.-H. Glassmeier, and W. S. Kurth (2004), Energetic electrons in the inner part of the Jovian magnetosphere and their relation to auroral emissions, J. Geophys. Res., 109, A06203, doi:10.1029/2004JA010405.

Tseng, W.-L., R. E. Johnson, and W.-H. Ip (2013), The atomic hydrogen cloud in the saturnian system, Planet. Space Sci., 85, 164-174, doi:10.1016/j.pss.2013.06.005.

Tsuchiya, F., H. Misawa, K. Imai, and A. Morioka (2011), Short-term changes in jupiter's synchrotron radiation at $325 \mathrm{mhz}$ : Enhanced radial diffusion in jupiter's radiation belt driven by solar uv/euv heating, J. Geophys. Res. (Space Physics), 116(A9), doi:10.1029/ 2010JA016303.

Van Allen, J. A., M. F. Thomsen, and B. A. Randall (1980), The energetic charged particle absorption signature of Mimas, Journal Geophys. Res., 85, 5709-5718, doi:10.1029/ JA085iA11p05709.

van Allen, J. A., B. A. Randall, and M. F. Thomsen (1980), Sources and sinks of energetic electrons and protons in Saturn's magnetosphere, Journal Geophys. Res., 85, 5679-5694, doi:10.1029/JA085iA11p05679.

Walt, M. (1994), Introduction to geomagnetically trapped radiation, 1st ed., Cambridge University Press, Cambridge, United Kingdom.

Williams, D. J., R. W. McEntire, S. Jaskulek, and B. Wilken (1992), The Galileo Energetic Particles Detector, Space Sci. Rev., 60, 385-412, doi:10.1007/BF00216863.

Wilson, R. J., R. L. Tokar, and M. G. Henderson (2009), Thermal ion flow in Saturn's inner magnetosphere measured by the Cassini plasma spectrometer: A signature of the Enceladus torus?, Geophys. Res. Lett., 36, 23,104, doi:10.1029/2009GL040225.

Wilson, R. J., F. Bagenal, P. A. Delamere, M. Desroche, B. L. Fleshman, and V. Dols (2013), Evidence from radial velocity measurements of a global electric field in saturn's inner magnetosphere, J. Geophys. Res. (Space Physics), doi:10.1002/jgra.50251.

Wilson, R. J., F. Bagenal, and A. M. Persoon (2017), Survey of thermal plasma ions in Saturn's magnetosphere utilizing a forward model, Journal of Geophysical Research (Space Physics), 122, 7256-7278, doi:10.1002/2017JA024117.

Woodfield, E. E., R. B. Horne, S. A. Glauert, J. D. Menietti, and Y. Y. Shprits (2013), Electron acceleration at jupiter: input from cyclotron-resonant interaction with whistler-mode chorus waves, Annales Geophysicae, 31(10), 1619-1630, doi:10.5194/ angeo-31-1619-2013. 
Woodfield, E. E., R. B. Horne, S. A. Glauert, J. D. Menietti, and Y. Y. Shprits (2014), The origin of Jupiter's outer radiation belt, J. Geophys. Res., 119, 3490-3502, doi:10.1002/ 2014JA019891.

Yoshikawa, I., K. Yoshioka, G. Murakami, A. Yamazaki, F. Tsuchiya, M. Kagitani, T. Sakanoi, N. Terada, T. Kimura, M. Kuwabara, K. Fujiwara, T. Hamaguchi, and H. Tadokoro (2014), Extreme ultraviolet radiation measurement for planetary atmospheres/magnetospheres from the earth-orbiting spacecraft (extreme ultraviolet spectroscope for exospheric dynamics: Exceed), Space Science Reviews, 184(1), 237-258, doi: 10.1007/s11214-014-0077-z.

Young, S. L., R. E. Denton, B. J. Anderson, and M. K. Hudson (2008), Magnetic field line curvature induced pitch angle diffusion in the inner magnetosphere, J. Geophys. Res., 113, A03210, doi:10.1029/2006JA012133. 